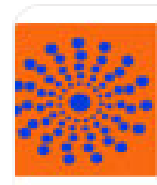

JOURNAL OF BEHAVIOR. healti \& SOCIAL issur

imis

Journal of Behavior, Health \& Social Issues

ISSN: 2007-0780

jcpedro@unam.mx

Asociación Mexicana de Comportamiento y

Salud, A. C.

México

Mazadiego-Infante, Teresa de Jesús; Vera-Pedroza, Alejandro; Ruiz-Carús, Saralina Problemas internalizados y externalizados en una muestra de niños de Educación Básica Journal of Behavior, Health \& Social Issues, vol. 3, núm. 1, mayo-octubre, 2011, pp. 17-23 Asociación Mexicana de Comportamiento y Salud, A. C.

Distrito Federal, México

Disponible en: http://www.redalyc.org/articulo.oa?id=282221799002

Cómo citar el artículo

- Número completo

- Más información del artículo

Página de la revista en redalyc.org

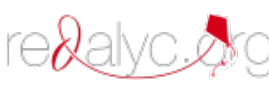

Sistema de Información Científica

Red de Revistas Científicas de América Latina, el Caribe, España y Portugal Proyecto académico sin fines de lucro, desarrollado bajo la iniciativa de acceso abierto 


\title{
PROBLEMAS INTERNALIZADOS Y EXTERNALIZADOS EN UNA MUESTRA DE NIÑOS DE EDUCACIÓN BÁSICA
}

\author{
INTERNALIZED AND EXTERNALIZED PROBLEMS \\ IN A SAMPLE OF ELEMENTARY SCHOOL CHILDREN
}

\author{
Teresa de Jesús Mazadiego-Infante \\ Facultad de Psicología, Campus Poza Rica, \\ Universidad Veracruzana, Veracruz, México
}

\author{
Alejandro Vera-Pedroza \\ Facultad de Pedagogía, Campus Poza Rica, \\ Universidad Veracruzana, Veracruz, México \\ Saralina Ruiz-Carús \\ 3Facultad de Psicología, Campus Poza Rica, \\ Universidad Veracruzana, Veracruz, México \\ ReCIBIDO: 5 DE ABRIL, 2010 \\ ACEPTADO: 24 DE FEBRERO, 2011
}

\section{Resumen}

Betancourt (2007) señala que los problemas internalizados son más difíciles de detectar, ya que el comportamiento de los sujetos no afecta de manera directa a los padres y maestros, subestimándose la gravedad de dichos problemas. Etienne (2005), Mazadiego (2005) y Pelish (2006) afirman que una baja autoestima puede llevar a una persona a tener estos problemas, mientras que una buena autoestima puede hacer que tenga confianza en las propias capacidades, no se deje manipular, sea más sensible a las necesidades del otro y esté dispuesto a defender sus principios y valores. El objetivo de la investigación fue disminuir los problemas que presentaba una muestra de niños reportados por sus profesores y canalizados a la Facultad de Psicología, Campus Poza Rica, de la Universidad Veracruzana. Participaron 100 niños de una escuela básica pública, en los grados de $4^{\circ}, 5^{\circ}$ y $6^{\circ}$ de primaria que presentaban conducta agresiva con sus condiscípulos, bajas calificaciones, minusvalía, conducta desafiante con sus profesores, desobediencia, bajo autoconcepto y baja autoimagen. El criterio de la muestra fue con base en lo reportado por los profesores de grupo de los tres grados. El instrumento utilizado fue la Escala de Autoestima de Fernández y Andrade (2007), la cual presentó una fiabilidad de 0.640. Los resultados indicaron que el programa de autoestima trabajado fue efectivo para todos los participantes, siendo el incremento más alto el de la autoestima en los varones, mejorando principalmente la autoestima con los amigos (área social), en la familia y en la escuela; en las tres áreas trabajadas hubo cambios sustanciales, por lo que se considera que el Programa de Autoestima utilizado fue eficaz en la disminución de los problemas que presentaban los participantes, aunque se recomienda un seguimiento para medir el impacto del programa.

Palabras clave: Problemas internalizados, problemas externalizados, programa de autoestima. 
Mazadiego-Infante, Vera-Pedroza \& Ruiz-Carús

Abstract

Betancourt (2007) indicates that internalized problems are more difficult to detect because subjects behavior does not affect directly their parents and teachers, who underestimate the severity of them. Etienne (2005), Mazadiego (2005) and Pelish (2006) argue that low self-esteem can lead a person to show these problems; on the contrary, a good self-esteem can make subjects confident in their abilities, to prevent to be manipulated, to be more sensitive to the needs of others, and to be willing to defend their principles and values. This research was designed to reduce the problems presented by a sample of children reported by their teachers and forwarded to the College of Psychology, Campus Poza Rica, Universidad Veracruzana. Participants were one hundred children that attended a public elementary school, in grades fourth, fifth and sixth of elementary level; they showed aggressive behavior with peers, poor grades, disabilities, challenging behavior with teachers, disobedience, low self-concept and low self-image. The criterion to be included in the sample was based on reports from the teachers of the three degrees. The instrument used was the Self-Esteem Scale of Andrade and Fernández (2007) which has a reliability of 0.640. Results indicated that the self-esteem program was effective for all participants, with the highest increase in male self-esteem; it was best in improving self-esteem, primarily with friends (social area) and also at home and at school; in the three areas of the program there were substantial changes, so it is considered that the self-esteem program was effective in reducing the problems of participants; further monitoring is recommended to measure the overall impact of the program.

Key words: Internalizing problems, externalizing problems, self-esteem program.

\section{Introducción}

Evidencias de investigaciones recientes realizadas en Latinoamérica y el Caribe por Maddaleno, Morello e Infante-Espínola (2003) permiten subrayar la importancia de realizar programas de intervención en niños y adolescentes, reportando que entre 11 y $33 \%$ de los adolescentes tienen problemas de depresión e intento de suicidio. Caraveo, Colmenares y Martínez (2002) detectaron que $16 \%$ de niños y adolescentes cuyas edades oscilan entre 4 y 16 años presentaron algún tipo de trastornos, entre los cuales destacaban inquietud, miedo, irritabilidad y nerviosismo. Tremblay, Mass, Pagani y Vitaro (1996) han señalado que sin intervención temprana los problemas emocionales y conductuales en menores de edad se pueden convertir en patrones de comportamientos, iniciando una trayectoria de problemas académicos, abuso de sustancias, delincuencia y violencia.

Para Molina, Tomas, Sabaté y Baeza (2001) existen bases para establecer dos amplias categorías de caracterización en niños y adolescentes: a) trastornos o desórdenes de interiorización que se describen como ansiedad, timidez, retraimiento y depresión, y b) trastornos o desórdenes exteriorizados, que se denominan desobediencia y destructividad. Después de haber realizado diferentes investigaciones relacio- nadas con problemas internalizados y externalizados en niños mexicanos, Betancourt (2007) señaló que los problemas internalizados son más difíciles de detectar, ya que el comportamiento de los sujetos no afecta de manera directa a los padres y maestros, y por lo tanto éstos subestiman la gravedad de dichos problemas.

Betancourt (2007), Fernández (2005) y Kuri (2008) encontraron que los problemas internalizados correlacionaron positivamente con la vergüenza en los niños estudiados, obteniendo mayor frecuencia la vergüenza con altos puntajes en problemas internalizados y externalizados. Las investigaciones acerca de los problemas internalizados y externalizados han demostrado que son diversos los factores que contribuyen al desarrollo de estos cuadros. Uno de estos factores es la autoestima, y eso se puede entender por la relación que tiene la autoestima con problemas como la depresión, la anorexia, la timidez, el abuso de drogas y otros. Se ha demostrado que una buena autoestima permite la autorregulación de la conducta (Branden, 1999, 2001; Markus \& Kunda, 1986). Gracias a ella se logra que los individuos tengan confianza en sí mismos y en sus capacidades, acepten retos en forma positiva y no se dejen manipular por los demás (Etienne, 2005; Pelish, 2006). Aunque se ha tratado de institucionalizar la enseñanza de la autoestima en las escuelas primarias y secundarias 
Problemas en niños de educación básica

(Villar, Luengo, Gómez \& Romero, 2003), ha sido difícil implementarla en la práctica.

La autoestima, a pesar de que reúne un conjunto de características y actitudes internas, se manifiesta y se desarrolla habitualmente dentro del contexto de la vida social tanto de la familia como de la escuela y el trabajo. La autoestima no pueda ser considerada simplemente como un sentimiento de autosatisfacción, debido a la gran relevancia que tiene en las relaciones con los demás y con el entorno, así como con el significado sobre la propia valía, lo que implica ser responsable hacia sí mismo y hacia los demás (Etienne, 2005; Mazadiego, 2005; Pelish, 2006).

La autoestima se caracteriza por ser un componente evaluativo y se relaciona con una variedad de situaciones. Se modifica a lo largo del desarrollo del niño y se adquiere a partir de la experiencia y de la interacción social que logre desarrollar (Burns, 1998). Asimismo, el autoconcepto tiene una dimensión social y se aprende como consecuencia de la experiencia al interactuar con el mundo físico y las personas significativas (Bandura \& Schunk, 1981; Lackovic-Grgin \& Dekovic, 1990). Para Frías, Mestre y Del Barrio (1994), el estilo de crianza de los padres durante los primeros tres o cuatro años determina la cantidad de autoestima inicial en los niños, y el control y apoyo parental que reciban están íntimamente relacionados con los problemas internalizados y externalizados de los niños en la etapa adolescente (Betancourt, 2007; Kuri, 2008).

El objetivo de la presente investigación fue modificar la autoestima en una muestra incidental de niños de educación básica para disminuir tanto sus problemas internalizados como externalizados.

\section{Método}

\section{Participantes}

Participaron 100 niños de una escuela primaria pública, 58 varones y 42 niñas, en los grados de $4^{\circ}, 5^{\circ}$ y $6^{\circ}$ de primaria que presentaban conducta agresiva con sus condiscípulos, bajas calificaciones, minusvalía, conducta desafiante, desobediencia, bajo autoconcepto y baja autoimagen. El criterio de la selección de la muestra fue con base en lo reportado por los profesores de cada uno de los tres grados. Antes de iniciar la intervención se les aplicó a los alumnos la Escala de Autoestima de Fernández y Andrade (2007) para confirmar el criterio de selección.
Instrumentos

Se utilizó la Escala de Autoestima de Fernández y Andrade (2007), la cual consta de 22 preguntas con tres posibles respuestas tipo Likert: a) no, b) algunas veces y c) siempre. La escala midió tres áreas: su autoestima en la escuela, en la familia y con sus amigos.

\section{Procedimiento}

Se hizo una reunión con los maestros y el director la escuela para explicarles en qué consistiría el trabajo de intervención, además de entregar a cada maestro de grupo la solicitud escrita para que los padres de familia autorizaran la participación de cada uno de sus hijos en el trabajo terapéutico. Dicha autorización debía estar firmada por los padres para que los alumnos pudieran ser registrados como participantes. A los niños se les aseguró que todos los comentarios que se dijeran durante las sesiones serían estrictamente confidenciales y que en el momento en que ya no quisieran participar eran libres de abandonar el aula sin perjuicio o regaño por parte de los maestros. Solamente hubo dos deserciones, ambas entre las niñas. La Variable Independiente fue el Programa de Autoestima y la Variable Dependiente fue el conjunto de las respuestas de cada uno de los participantes en un diseño preexperimental antes-después con cuatro grupos experimentales y cuatro grupos de control: todos los niños seleccionados por los profesores fueron agrupados por edades, sirviendo de grupo control de cada grado el resto de los condiscípulos en cada grado que no fueron seleccionados.

Cada grupo experimental estuvo conformado por 25 niños y se les asignó un día a la semana para trabajar durante dos horas. Hubo una etapa de sensibilización para integrarlos y establecer un clima de confianza, además de la lectura obligada de carteles colocados en la pared con la leyenda Lo que aquí se dice, aquí se queda, explicándoles el significado. La edad de los niños estuvo comprendida en un rango de 10 a 13 años, del cuarto al sexto grado de educación básica de una escuela primaria pública, de nivel socioeconómico medio-bajo. La aplicación tuvo lugar en un salón de clase destinado para el trabajo grupal de las diversas dinámicas utilizadas en cada dimensión del programa de autoestima de la propia investigadora. Los materiales fueron: CDs de música de relajación, estrategias terapéuticas humanistas, carteles, hojas blancas, crayolas y colchonetas. Se trabajó el autoconocimiento, el autoconcepto, la au- 
toevaluación, la autoaceptación, el autorrespeto y la autoestima. Cada aspecto se trabajó desde la Teoría Humanista en cuatro sesiones en cada grupo, y se trataron las áreas familiar, escolar y social. Se solicitó que los participantes mencionaran los problemas más frecuentes por los cuales eran castigados o que les generaban problemas en la escuela, en la familia y con sus amigos. Se realizaron dinámicas grupales para cada tema, se compartieron experiencias y posteriormente ellos propusieron alternativas para mejorar y disminuir sus problemas internalizados.

Resultados

La Escala de Autoestima mostró una fiabilidad total de 0.640, obtenida a través de la prueba alfa de Cronbach. Se analizó la autoestima en forma global antes $(\mathrm{t}=74.02, \mathrm{p}<0.001 ; \mathrm{M}=30.0714 ; \mathrm{S}=2.88)$ y después $(t=103.24, p<0.001 ; M=36.7640 ; S=$ 4.68). A través de la comparación de las medias se observó una diferencia significativa de 6.69, lo que indicó un incremento en la autoestima de los participantes en forma grupal. A la pregunta de investigación: ¿Qué tan efectiva será la propuesta de autoestima para disminuir los problemas internalizados y externalizados de una muestra incidental de niños de educación básica?, los resultados responden favorablemente, pues se identifica un incremento significativo de la autoestima de los participantes y un decremento de los problemas externalizados. Al final de la intervención los participantes comunicaron sentirse mejor al no ser castigados, al llevarse mejor con los amigos y compañeros de clase, al elevar sus calificaciones e incluso al ser premiados por algunos padres de familia que reportaron a sus hijos más obedientes.

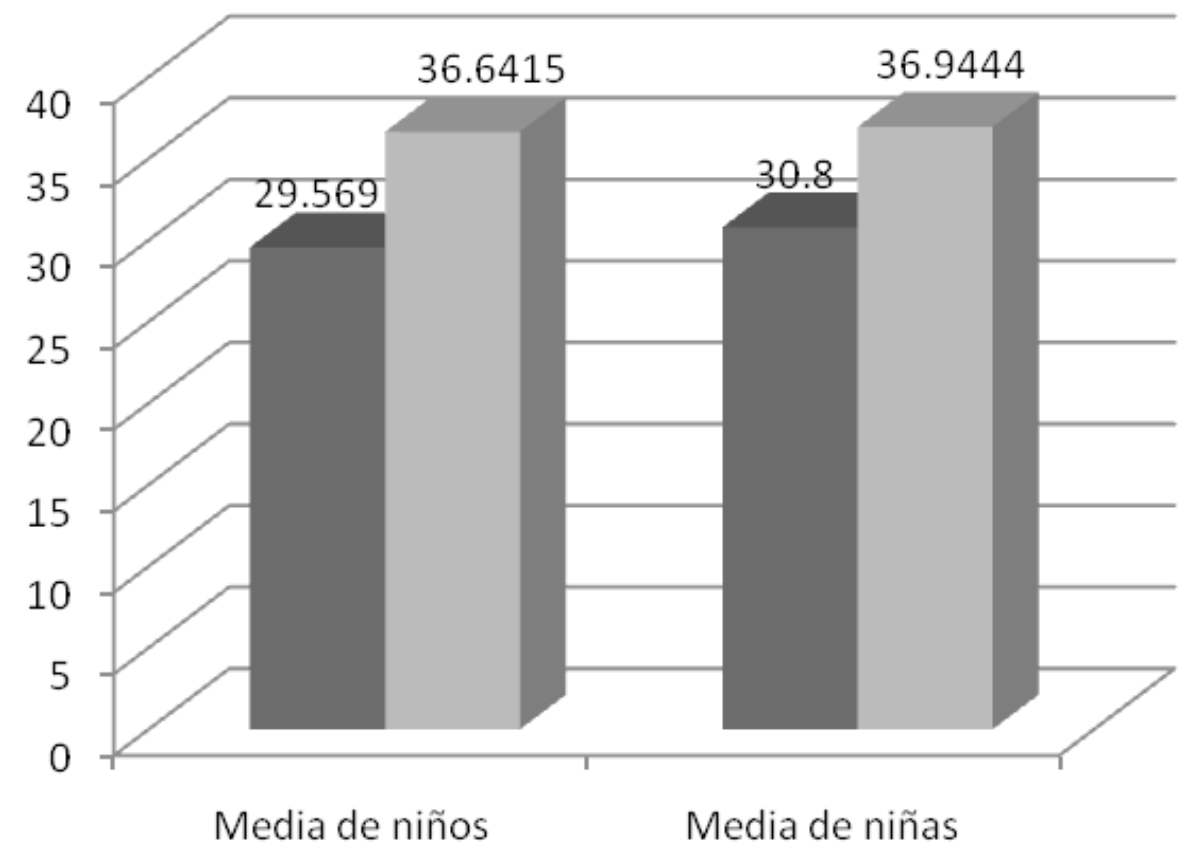

preprueba

postprueba

Figura 1. Comparación de la media de la autoestima antes y después de la intervención en niños y niñas. 
Problemas en niños de educación básica

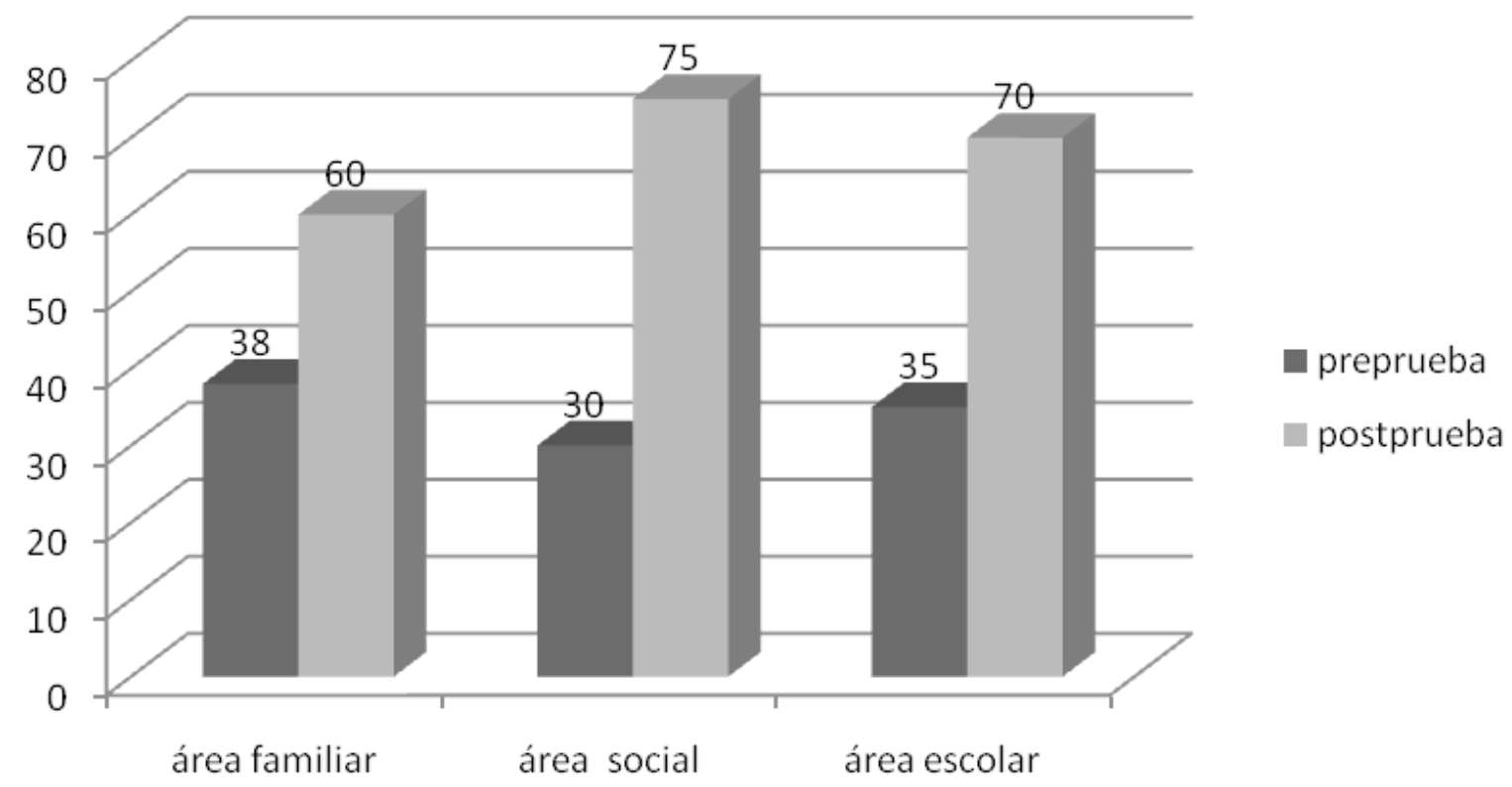

Figura 2. Comparación de las puntuaciones obtenidas en la Escala de Fernández y Andrade (2007) sobre las áreas trabajadas antes y después de la intervención terapéutica.

Comparando los grupos de niños y niñas se notaron cambios significativos en las medias, siendo el cambio en la autoestima mayor en las medias de los niños $(M=7.08)$.

En el área donde destacó más el incremento de la autoestima fue con los amigos, pues las conductas agresivas disminuyeron, notándose más amistosos; en el área escolar las conductas desafiantes disminuyeron visiblemente, las calificaciones aumentaron en todos los participantes, describiendo un autoconcepto de sí mismos diferente al inicial del programa, y su autoimagen también se incrementó; en el área familiar los padres notaron un cambio sustancial en la obediencia de sus hijos.

\section{Discusión}

El objetivo de la investigación fue alcanzado, pues fue posible disminuir los problemas internalizados, lo cual se vio reflejado en conductas positivas y, por lo tanto, los problemas externalizados como la agresividad, la conducta desafiante y la desobedien- cia (Betancourt, 2007; Fernández, 2005; Kuri, 2008) disminuyeron notablemente.

El trabajo de la autoestima contribuyó a establecer una relación positiva de los niños con sus condiscípulos. Los niños se mostraban con mayor seguridad, alegres, juguetones, obedientes, dispuestos a poner atención y más optimistas, siendo un dato rescatable su aceptación de "ser como son" y no estar esperando a llenar expectativas de los adultos que los frustraban; el hablarlo en las sesiones permitió su crecimiento personal.

Estudios referidos a la psicopatología del desarrollo han demostrado que si no se atienden a edad temprana los problemas externalizados de los niños, algunos trastornos iniciados en esta etapa persisten hasta la edad adulta y se asocian con otros trastornos psiquiátricos (Caraveo, Colmenares \& Martínez, 2002), por lo que la pregunta de investigación: ¿Qué tan efectiva será la propuesta de autoestima para disminuir los problemas internalizados y externalizados de una muestra incidental de niños de educación básica?, fue respondida eficazmente. Los resultados muestran 
un decremento de las conductas de agresividad, un incremento en el autoconcepto, en su autoimagen y en su autoestima, impactando positivamente sobre las calificaciones de todos los participantes.

El control que ejercen los padres sobre el niño y el apoyo parental que le ofrezcan está altamente relacionado con los problemas internalizados y externalizados que muestren los niños (Aguilar, 2002; Betancourt, 2007; Kuri, 2008), y aunque en este trabajo de investigación los padres participaron poco, el impacto del estilo de crianza y el control que ejercen sobre sus hijos tiene fuerte relación con una autoestima alta o baja, según sea el control parental ejercido. Por otra parte, Markus y Kunda (1986) han señalado que al no mantener un adecuado control parental surge el rechazo paternal, exteriorizando ausencia de calor, afecto o amor de los padres hacia los hijos o privándolos de ello. Por esta razón Achenbach y Edelbrock (1981) y Harvey (1991) mencionan que la presencia de problemas emocionales y de conducta durante la niñez permite predecir futuros problemas de adaptación durante la adolescencia y la edad adulta, de manera que es conveniente identificarlos a edades tempranas.

Se ha reportado que en los centros escolares donde se presentan problemas de conducta como la agresión, el bajo rendimiento escolar, los delitos de robo menores, etcétera, existen niños o niñas silenciosos que exhiben una actitud depresiva, insegura o nerviosa, lo que impide un desarrollo emocional saludable. Debido a lo anterior, es importante institucionalizar la enseñanza de la autoestima en las escuelas primarias y secundarias por las relaciones que se establecen con los demás y con el entorno. El éxito de esta estrategia permitirá encontrar significado de la propia valía, implicando ser responsable hacia sí mismos y hacia los demás, logrando seguridad en sí mismos y no dejarse manipular (Bandura \& Schunk, 1981; Lackovic-Grgin \& Dekovic, 1990; Maddaleno, Morello \& Infante-Espínola, 2003; Mazadiego, 2005; Villar, Luengo, Gómez \& Romero, 2003).

La Escala de Autoestima para niños de Fernández y Andrade (2007) permitió conocer la autoestima desarrollada con los amigos, en la escuela y en la familia, donde se notaron cambios significativos. De cualquier manera, se sugiere realizar mayores estudios para la confirmación de los resultados encontrados.

En conclusión, los resultados indicaron que el programa de autoestima trabajado fue efectivo para todos los participantes, siendo el incremento de autoestima más alto entre los varones, mejorando principalmente la autoestima con los amigos (área social), en segundo lugar en la familia y por último en la escuela. Sin embargo, en las tres áreas trabajadas hubo cambios sustanciales, por lo que se considera que el Programa de Autoestima utilizado fue eficaz en la disminución de los problemas que presentaban los cien participantes. Ahora bien: a pesar de los cambios sustanciales observados, es recomendable realizar un seguimiento seis meses después de aplicado el programa para medir el impacto del mismo y proponer nuevas estrategias de intervención. Además es recomendable observar la interrelación familiar.

\section{Referencias}

Achenbach, T., \& Edelbrock, C. (1981). Behavioral problems and competencies reported by parents of normal and disturbed children aged four through sixteen. Monographs of the Society for Research in Child Development, 188-193.

Aguilar, E. (2002). Familias con autoestima. México: Trillas.

Bandura, A., \& Schunk, D. H. (1981). Cultivating competence, self efficacy, and intrinsic interest through proximal self-motivation. Journal of Personality and Social Psychology, 586-598.

Branden, N. (2001). El poder de la autoestima. México: Paidós.

Branden, N. (1999). Los seis pilares de la autoestima. Madrid: Editorial Paidós.

Betancourt, D. (2007). Control parental y problemas internalizados y externalizados en niños y adolescentes. Tesis Doctoral inédita. México: UNAM.

Burns, R. B. (1998). El autoconcepto. Teoría, medición, desarrollo y comportamiento. Bilbao: EGA.

Caraveo, J. Colmenares, E., \& Martínez, N. (2002). Síntomas, percepción y demanda de atención en salud mental en niños y adolescentes de la Ciudad de México. Salud pública de México, 44, (6). Recuperado el 11 de agosto de 2011 de: http://redalyc.uaemex.mx/redalyc/src/inicio/ ArtPdfRed.jsp?iCve=10644601

Etienne, A. (2005). La autoestima en el sistema teórico de Rogers. Recuperado el 11 de agosto de 2011 de: http://www.cop.es/colegiados/GR00724/ art\%C3\%ADculo\%20autoestima\%20Rogers.doc 
Problemas en niños de educación básica

Fernández de O., B.M. (2005). La vergüenza en los niños, su conceptualización psicológica, medición y sus correlatos conductuales. Tesis doctoral inédita. Facultad de Psicología, México: UNAM.

Fernández, R., \& Andrade, P. P. (2007). Escala de autoestima en niños. México: UNAM.

Frías, D., Mestre, V., \& Del Barrio, V. (1994). Autoestima y depresión en niños. Revista de Psicología General y Aplicada, 47, 471-476.

Harvey. V. S. (1991). Characteristics of children referred to school psychologists: A discriminant analysis. Psychology in the Schools, 28, 209-218.

Kuri, P.V. (2008). Relación del control y apoyo parental con los problemas internalizados y externalizados de adolescentes de secundaria. Tesis de Licenciatura inédita. Veracruz, México: Universidad Veracruzana.

Lackovic-Grgin, K., \& Dekovic, M. (1990). The contribution of significant others to adolescents' self-esteem. Adolescence, 25, 839-846.

Maddaleno, M, Morello P., \& Infante Espinola, F. (2003). Salud y desarrollo de adolescentes y jóvenes en Latinoamérica y el Caribe: desafíos para la próxima década. Salud Pública de México, 45, suplemento 1, 132-139.
Markus, H., \& Kunda, Z. (1986). Stability and malleability in the self-concept in the perception of others. Journal of Personality and Social Psychology, 51, 181-190.

Mazadiego, I.T. (2005). Propuesta humanista para la clarificación de valores en estudiantes universitarios. Tesis doctoral, Universidad Iberoamericana, Santa Fe: Biblioteca Javier Clavijero.

Molina, M., Tomas, J., Sabaté, N., \& Baeza, E. (2001). Trastornos depresivos en niños y adolescentes. Problemáticas de la adolescencia y otros aspectos de la edad evolutiva. Barcelona: Laertes.

Pelish, P. (2006). Effects of a self-esteem intervention program on school-age children. Pediatric Nursing 32, 341-348.

Tremblay, R. E., Mass, L. C., Pagani, L. \&, Vitaro, F. (1996). From childhood physical aggression to adolescent maladjustment: The Montreal prevention experiment. Preventing childhood disorders, substance abuse and delinquency. Thousand Oaks, CA, EUA: Sage Publications Inc.

Villar, P., Luengo, M., Gómez, J., \& Romero, E. (2003). Una propuesta de evaluación de variables familiares en la prevención de conductas problema en la adolescencia. Psicothema, 15, 581-588. 